\title{
The term structure of cross-sectional dispersion of expectations in a Learning-to-Forecast Experiment
}

\author{
Annarita Colasante, Simone Alfarano, Eva Camacho-Cuena
}

\begin{abstract}
In this paper, we present the results of a Learning-to-Forecast Experiment (LtFE) where we eliciting short- as well as long-run expectations regarding the future price dynamics in markets with positive and negative expectations feedback. Comparing our results on short-run expectations with the LtFE literature, we prove that eliciting long-run expectations has no impact on the price dynamics nor on short-run expectations formation. In particular, we confirm that the Rational Expectation Equilibrium (REE) is a good benchmark only for the markets with negative feedback. Interestingly, our data show that while the term structure of the cross-sectional dispersion of expectations is convex in positive feedback markets, it is concave in negative feedback markets. Differences in the slope of the term structure stem from diverse degrees of uncertainty regarding the evolution of prices in the two feedback systems: (i) in the negative feedback system, the convergence of the price to the REE reflects a tendency for coordination of long-run expectations around the fundamental value; (ii) conversely, oscillatory price dynamics observed in the positive feedback system is responsible for the diverging pattern of long-run expectations. Finally, we propose a new measure of heterogeneity of expectations based on the scaling of the dispersion of expectations over the forecasting horizon.

keywords: Long-Run Expectations; Heterogeneous Expectations; Experiment; Coordination; Convergence; Learning-to-Forecast Experiment. JEL: D03, G12, C91
\end{abstract}




\section{Introduction}

The expectations of an economic agent regarding the future state of the economy affects his/her current individual choices. Thus, when aggregating all individual choices, expectations of agents influence the realizations of macroeconomic quantities. At the same time, the evolution of macroeconomic aggregates has an impact on how agents form and revise their expectations. The economy can therefore be modelled as an expectations feedback system. Consequently, how agents form their expectations at the individual level plays an important role in understanding the dynamics of the aggregate outcome. The rational expectations framework provides a normative indication of how expectations should be consistently formed within a given model. In the framework of rational expectations, agents share a common expectations formation mechanism. In order to introduce heterogeneity within the rational expectations framework, Mankiw et al. (2003) propose a sticky information model, where they assume that agents follow rational expectations and update their information set at different moments, taking into account acquisition and computation costs. Therefore, the heterogeneity of expectations emerges from the agents' heterogeneous information set. We find, however, numerous examples in the theoretical, computational and experimental literature that rely on bounded rationality to introduce heterogeneity in the formation of the agents' expectations (see Hommes (2013) and references therein).

The origin of heterogeneity across individual expectations and the role that it plays in shaping aggregate outcomes is an important topic in theoretical as well as empirical research in macroeconomics. The fact that expectations are not directly observable in the same way as prices or volumes, means that there is a significant limitation when it comes to fully understanding their precise role in driving macroeconomic aggregates.

Conducting forecasting surveys is one of the traditional methods used to elicit individual expectations (see Manski (2004)). This methodology has been extensively used in macroeconomics, e.g. in testing the accuracy and rationality of forecasters. Only recently has disagreement about expectations, measured as the cross-sectional dispersion of forecasts, and its evolution over time, become in itself a variable of interest. It seems to contain information about, for example, the uncertainty about future development of business cycles or inflation rates (see Mankiw et al. (2003)). In order to disentangle whether the origin of the observed forecast' dispersion is a measure of the intrinsic uncertainty of macroeconomic variables or if it reflects heterogeneous priors of forecasters, Patton and Timmermann (2010) focus on how the dispersion of expectations evolves over different time horizons. They study 
the term structure of cross-sectional dispersion of forecasts, observing that it typically increases with the forecast horizon. Their analysis reveals that such persistent heterogeneity of expectations among forecasters in the short-run stems from different private information, whereas in the long-run it is due to the forecasters heterogeneity regarding their prior and/or prediction models.

Laboratory experiments, such as surveys, allows us to directly elicit individual expectations, with the additional advantages of monitoring the information available to the subjects and using performance-based incentives. Within the experimental literature, LtFEs, introduced by Marimon et al. (1993), make it possible to study the formation of individual expectations within different expectations feedback systems, where the price depends on subjects' predictions. By using this experimental framework, many experiments investigate the expectations of agents in financial markets (Hommes et al. (2005), Bao and Ding (2016)), commodity markets (Bao et al. (2013)) and aslo in a macroeconomic framework (Assenza et al. (2011), Cornand and M'baye (2016)), to cite just a few contributions. In these articles, subjects have to predict prices within a 1 or 2 step-ahead horizon. One of the most pervasive results is that subjects show a certain degree of heterogeneity in their predictions, which seems to decrease over time. However, the reported level of heterogeneity is based on subjects' short-run predictions only, which might distort the evaluation of their level of disagreement.

In order to explain the disagreement among subjects' predictions, the literature on LtFEs argued that subjects follow heterogeneous anchor-andadjustment rules, anchoring their expectations to past prices. ${ }^{1}$ The dynamics of prices depends crucially on the expectations feedback system, showing large oscillations around the fundamental value under a positive feedback system and a fast convergence to the fundamental value under a negative feedback system. We might think that the evolution of the level of disagreement among subjects over time and across multiple forecast horizons is directly affected by the price dynamics observed under the two feedback systems.

Although there is an extensive macroeconomic literature that measures the term structure of forecasts using survey data to study the disagreement in expectations, the empirical characterization of the term structure of expectations in LtFEs is still missing. The aim of our paper is to investigate the impact of the expectations feedback system on the formation of long-run

\footnotetext{
${ }^{1}$ Anufriev and Hommes (2012) and Hommes (2013) introduce an heterogeneous expectations model showing that a set of different anchor-and-adjustment rules can successfully reproduce experimental data in a LtFE eliciting short-run expectations. Colasante et al. (2018a,b) show that subjects do indeed anchor their short- and long-run expectations to the last realized price in a positive feedback system.
} 
expectations as well as the empirical characterization of the term structure of subjects' predictions. The term structure analysis allows us to have a more precise idea regarding the level of disagreement among subjects. In fact, if we do not consider a broader spectrum of expectations, we could lead us to underestimate or overestimate subjects' disagreement. As an example, let us consider a scenario where subjects exhibit a strong coordination of their short-run forecasts together with an increasing dispersion of their long-run expectations. Measuring subjects' disagreement as the variance of their short-run forecasts would lead us to underestimate the level of disagreement among subjects. On the contrary, we can imagine a situation where a higher dispersion of short-run forecasts, compared to the first scenario, remains constant or decreases over different forecast horizons (constant or decreasing term structure). In this case, we may overestimate subjects' disagreement. We take the position that the term structure of expectations provides crucial information when it comes to characterizing the heterogeneity of expectations in LtFEs, and therefore long-run expectations cannot be ignored.

We conduct a LtFE in which, unlike the standard settings ${ }^{2}$, subjects submit their predictions for periods ranging from 1 to more than 10-step-ahead time horizon. We elicit subjects' long-run expectations at the beginning of every period, making it possible to revise their expectations as new information becomes available. In particular, we extend the novel setting introduced by Colasante et al. (2018a) and compare two different expectations feedback systems. As in Heemeijer et al. (2009), we compare two treatments: one with a positive feedback system and the other with a negative one. The positive feedback system mimics the behavior of financial markets where prices typically rise if investors expect positive changes. Conversely, the negative feedback system simulates commodity markets where, due to the delay in the production adjustment, market prices move in the opposite direction to expectations.

Our results on coordination and convergence of short-run expectations, as well as on the evolution of prices, are in line with those reported in the LtFEs literature (see Hommes (2013)). We propose a one-parameter term structure model to characterize the degree of disagreement of expectations in the two treatments. This characterization provides us with relevant information on how subjects form their long-run expectations. Extending the forecasting horizon reveals that subjects learn the REE in markets with negative feedback. We observe that the dispersion of long-run expectations around the

\footnotetext{
${ }^{2}$ For a comprehensive survey of macroeconomic experiments on expectations see Assenza et al. (2014).
} 
fundamental price decreases over time to reach almost full convergence to the REE. By estimating the term structure of expectations, we demonstrate that mutual coordination of expectations and their convergence to the fundamental price follow the same pattern. In contrast, in the market with positive feedback, we find a persistently high level of disagreement among forecasters and it is compatible with the heterogeneous extrapolative rules employed by subjects when forming their expectations.

The rest of the paper is organized as follows: section 2 describes the experimental setting, while section 3 introduces the theoretical framework and the working hypotheses. Section 4 presents the results of our empirical analysis, and finally, section 5 summarizes the main findings and concludes.

\section{Experimental Design}

We conduct a LtFE similar to Colasante et al. (2018a) where the subjects' task is to forecast the evolution of prices at different time horizons. In order to test the effect of the feedback system on expectations formation, we implement two treatments: in the first, we consider a market with positive feedback, while in the second treatment we consider a market with negative feedback between the expectations of subjects and the market price. ${ }^{3}$

Each market consists of six subjects playing the role of professional forecasters for 20 periods (see the translated instructions in Appendix B.1). Subjects are asked to submit their short- and long-run price predictions. This means that, at the beginning of period $t$, subject $i$ submits his/her short-run prediction for the market price at the end of period $t$, denoted as ${ }_{i} p_{t, t}^{e}$, as well as his/her set of long-run predictions for the price at the end of each one of the $20-t$ remaining periods. Long-run predictions are denoted as ${ }_{i} p_{t, t+k}^{e}$ with $1 \leq k \leq 20-t .{ }^{4}$

We implement the price adjustment mechanism proposed by Heemeijer et al. (2009) and employed by Colasante et al. (2018a) in a LtFE which elicits long-run expectations. In the positive feedback treatment the realized price depends positively on the average short-run price forecasts. The adjustment mechanism is as follows:

$$
p_{t}=p_{f}+\frac{1}{1+r}\left(\bar{p}_{t, t}^{e}-p_{f}\right)+\epsilon_{t} .
$$

\footnotetext{
${ }^{3}$ For the positive feedback treatment we use the dataset from Colasante et al. (2018a).

${ }^{4}$ Although within our experimental setting we collect 201 -step-ahead predictions from each subject instead of 50 as in most LtFEs, we can monitor the entire time-spectrum of expectations and its evolution over time.
} 
In the negative feedback treatment the realized price in each period depends negatively on the average subjects' short-run forecasts and is computed as follows:

$$
p_{t}=p_{f}-\frac{1}{1+r}\left(\bar{p}_{t, t}^{e}-p_{f}\right)+\epsilon_{t},
$$

where $r=0.05$ in all sessions. The constant fundamental price is computed as $p_{f}=\frac{d}{r}$ and $d$ is equal to 3.5 or 3.25 depending on the session. ${ }^{5}$ The term $\bar{p}_{t, t}^{e}$ in the pricing equations is the average of the six 1-step-ahead predictions submitted at the beginning of period $t, \bar{p}_{t, t}^{e}=\frac{1}{6} \sum_{i=1}^{6} p_{t, t}^{e}$. Finally, the term $\epsilon_{t} \sim N(0,0.25)$ is an iid normal shock. The main difference between eq. (1) and eq. (2) is how expectations affect the price: eq. (1) describes a positive feedback system where subjects predictions are self-fulfilling, i.e. the higher the average forecast is, the higher the price will be; eq. (2), on the other hand, describes a system in which there is a negative feedback between expectations and price.

Individual earnings in each period are computed as ${ }_{i} \pi_{t}={ }_{i} \pi_{t}^{s}+{ }_{i} \pi_{t}^{l}$ and depend on the accuracy of the subject's short- and long-run forecasts. We denote the profit depending on the accuracy of short- and long-run expectations as ${ }_{i} \pi_{t}^{s}$ and ${ }_{i} \pi_{t}^{l}$, respectively. Subjects' gains from their short-run predictions depend on a hyperbolic function of the quadratic forecasting errors:

$$
{ }_{i} \pi_{t}^{s}=\frac{250}{1+\zeta_{i, t}} \quad \text { with } \quad \zeta_{i, t}=\left(\frac{{ }_{i} p_{t, t}^{e}-p_{t}}{2}\right)^{2} .
$$

We define ${ }_{i} \pi_{t}^{l}=\sum_{j=1}^{t-1}{ }_{i} \pi_{t-j, t}^{l}$, where ${ }_{i} \pi_{t-j, t}^{l}$ represents the individual profit associated with the accuracy of the prediction submitted by subject $i$ at the beginning of period $t-j$ about the asset price in period $t$, where $1 \leq j \leq t-1$. It is computed according to the following payment schedule ${ }^{6}$ :

$$
{ }_{i} \pi_{t-j, t}^{l}=\left\{\begin{array}{l}
25 \quad \text { if } \quad 0 \leq{ }_{i} \delta_{t-j, t} \leq 5 \\
12 \quad \text { if } \quad 5<{ }_{i} \delta_{t-j, t} \leq 10 \\
5 \quad \text { if } \quad 10<{ }_{i} \delta_{t-j, t} \leq 15 \\
0 \quad \text { otherwise }
\end{array}\right.
$$

where ${ }_{i} \delta_{t-j, t}=\left|{ }_{i} p_{t-j, t}^{e}-p_{t}\right|$. The total profit of each subject is the sum of

\footnotetext{
${ }^{5}$ The values of the interest rate and average dividend are constant through a given session. To avoid the effects of communication among subjects between sessions, we set two different values of the dividend, so that we have some markets with a fundamental price of 65 and others with 70 .

${ }^{6}$ We used a payoff mechanism similar to Haruvy et al. (2007).
} 
profits across all periods. ${ }^{7}$ Note that we use a step-function instead of a smooth payoff function similar to eq. (3) to compute the profit for longrun predictions. Whereas subjects receive an immediate feedback on the forecasting errors of their short-run predictions, they experience a certain delay in evaluating the accuracy of their long-run predictions. Forecasting prices in the long-run is a more difficult task than forecasting short-run prices. It is for this reason that we consider that a step function guarantees to the subjects an easier evaluation of their long-run forecasting accuracy. ${ }^{8}$ In order to reward the short-run predictions of the subjects, we consider a hyperbolic function so as not to "force" subjects to coordinate. For small forecasting errors (i.e. $\zeta_{i, t}<1$ ), eq. (3) exhibits a parabolic shape as the function proposed by Hommes et al. (2004) and Heemeijer et al. (2009), while, for larger forecasting errors, it gradually approaches the zero lower bound of the payoff.

The information set available to the subjects when submitting their predictions is the following: the interest rate $(r)$, the average dividend $(d)$ (in the positive feedback treatment), the time series of prices up to period $t-1$ as well as all their own (short and long-run) past predictions. Profits are calculated at the end of each period and subjects receive information about the earnings of the last period together with the cumulative profit up to the current period. We provide qualitative information about the implemented feedback system, i.e. whether there is a positive or negative relationship between subjects' 1-step-ahead predictions and the realized price. Equations (1), (2) and (3) are not given to the subjects. To be sure that all subjects correctly understand the information provided in the instructions, the experimenter explains the instructions when presenting the software. During this presentation, subjects are informed that the market price is computed using their 1-step-ahead predictions. ${ }^{9}$ In order to give the subjects some reference point as to how short-term predictions are rewarded, they are informed that the maximum payoff for each 1-step-ahead prediction is $250 \mathrm{ECU}$ and that this amount falls with the forecasting error, reaching a value close to zero when the forecasting error is larger than 15.

\footnotetext{
${ }^{7}$ We assigned the same weight to short and long-run predictions in the subjects' final payoff by calibrating the parameters of the pay-off functions so that approximately $\max \sum_{t=1}^{20}{ }_{i} \pi_{t}^{s}=\max \sum_{t=1}^{20}{ }_{i} \pi_{t}^{l}$.

${ }^{8}$ We conducted additional sessions for negative as well as positive feedback treatments and implemented a rescaled hyperbolic continuous payoff function for both, long and shortrun predictions. Results are qualitatively and quantitatively similar to those described in the present paper (material available upon request).

${ }^{9}$ In the software, 1-step-ahead predictions (in green) have a different colour from longterm predictions(in black). A screenshot of the experiment is shown in Appendix B.2.
} 
The experimental sessions were conducted in the Laboratory of Experimental Economics at the Jaume I University. A total of 90 subjects $^{10}$ participated and 15 markets were implemented: 7 with positive feedback and 8 with negative feedback. Each session lasted approximatively 50 minutes and the average gain was of 20 Euros.

\section{$3 \quad$ Working Hypotheses}

Given the adjustment mechanism in eqs. (1) and (2), the REE predicts that the realized price $p_{t}$ converges to the fundamental value with fairly small fluctuations proportional to the idiosyncratic shock term $\epsilon_{t}$. If we assume that all subjects follow rational expectations, their predictions submitted in each period $t$ and for each forecast horizon $k$ fluctuate around the constant fundamental value irrespective of the expectations feedback system, i.e. ${ }_{i} p_{t, t+k}^{e} \approx p_{f}$. Plugging this condition into eqs. (1) and (2), we obtain $p_{t}=p_{f}+\epsilon_{t}$.

\subsection{Short-run predictions}

In our experimental setting, the REE does not depend on the expectations feedback system. On the contrary, as routinely identified in the LtFE literature, the feedback system plays a crucial role in whether and how prices converge to the REE and in the dynamics of the coordination of expectations. Following the general theoretical predictions of Haltiwanger and Waldman (1989), in the case of markets with positive feedback, subjects' short-run expectations can be considered as strategic complements. In these markets, therefore, subjects have a strong incentive to mutually coordinate their shortrun predictions although not necessarily with the REE. The convergence to the REE is indeed difficult to achieve, since it requires that (almost) all subjects' expectations are coordinated around the fundamental value. In markets with negative feedback, subjects' expectations can be considered as strategic substitutes, so that subjects have an incentive to predict low (high) prices when they expect that the other subjects will predict high (low) prices. Consequently, in these markets we expect to see a lower degree of coordination of subjects' short-run expectations. Moreover, the convergence to the REE is more likely to be observed, since it is sufficient for expectations to be (on average) close to the fundamental value, instead of the more stringent

\footnotetext{
${ }^{10}$ Each subject participated in only one session and had not previously taken part in LtFEs.
} 
conditions of the positive feedback, where (almost) all subjects' expectations should coordinate around $p_{f}$.

By means of LtFEs, Heemeijer et al. (2009) show that the dynamics of individual 1-step-ahead price predictions, as well as the dynamics of the realized price, largely depend on the particular nature of the expectations feedback system, which confirms the general theoretical predictions of Haltiwanger and Waldman (1989). In particular, Heemeijer et al. (2009) find that, in markets with positive feedback, subjects coordinate their individual one-step-ahead predictions in a few periods, while the realized price needs a much longer number of periods to achieve some degree of convergence to the fundamental value. On the contrary, under negative feedback, the realized price exhibits a fast convergence to the fundamental value, whereas subjects' predictions need a slightly higher number of periods to coordinate. Unlike markets with positive feedback, the coordination of short-run predictions is almost parallel to their convergence to the fundamental value.

Since our experimental setting shares the main characteristics of the experiment reported in Heemeijer et al. (2009), we expect to observe similar time series properties of prices and short-run predictions depending on the feedback system.

Hypothesis 1a: Markets with positive feedback are characterized by a coordination of short-run predictions within a few periods and a slow convergence of the realized price to the fundamental value.

Hypothesis 1b: Markets with negative feedback are characterized by a faster convergence of the realized price to the fundamental value with a simultaneous coordination of short-run predictions.

If Hypotheses $1 \mathrm{a}$ and $1 \mathrm{~b}$ are not rejected, we can argue that eliciting longrun expectations has no significant effect on the subjects' short-run predictions nor on realized price dynamics in the positive as well as in the negative expectations feedback system. ${ }^{11}$

\subsection{The term structure of the cross-sectional disper- sion of subjects' predictions}

When eliciting their long-run expectations, at the beginning of period $t$ subjects submit their price predictions for the end of period $t+k$, for all $k>0$.

\footnotetext{
${ }^{11}$ Colasante et al. (2018b) show that Hypothesis 1a cannot be rejected in the positive expectations feedback system.
} 
According to eqs. (1) and (2), the price at the end of period $t+k$ depends on the subjects' short-run predictions submitted at the beginning of period $t+k$. Therefore, each subject should guesstimate, $k$-periods in advance, the short-run expectations of the other subjects. We expect that long-run predictions exhibit a lower degree of coordination, or equivalently a higher degree of disagreement, the longer the forecast horizon is, given the increasing uncertainty in guesstimating the future short-run behavior of the other subjects.

In order to better characterize the level of disagreement among subjects by also considering its evolution over time under the different feedback systems, we analyze the term structure of the cross-sectional dispersion of subjects' predictions across different forecast horizons. Our conjecture is that the shape of the term structure is qualitatively different depending on the expectations feedback system. To illustrate more precisely our conjecture concerning the shape of the term structure of subjects' predictions, let us assume that subjects anchor their long-run expectations to the last realized price and linearly extrapolate the past price change. We assume that each subject has an ex-ante different prior on the extrapolation coefficient $m_{i}$, and, for simplicity, we consider that all coefficients are independent of the period and the forecast horizon. Coefficient $m_{i}$ can be interpreted as the strength of the trend extrapolation. ${ }^{12}$ The (short- and long-run) expectations formation rule can be formalized as follows:

$$
{ }_{i} p_{t, t+k}^{e}=p_{t-1}+m_{i}(k+1)\left(p_{t-1}-p_{t-2}\right),
$$

where $0 \leq k \leq K$, and $K$ is the maximum forecasting horizon. ${ }^{13}$ Note that, according to eq. (4), the only source of heterogeneity among subjects in the formation of expectations is directly linked to their different priors on $m_{i}$. The value of the variance of subjects' predictions for a given period and forecasting horizon is a proxy for their degree of disagreement. From eq. (4), it is possible to show that (see Appendix A for the calculation details.): ${ }^{14}$

$$
\operatorname{Var}\left[{ }_{i} p_{t, t+k}^{e}\right]=\operatorname{Var}\left[m_{i}\right](k+1)^{2}\left(p_{t-1}-p_{t-2}\right)^{2}=(k+1)^{2} \operatorname{Var}\left[{ }_{i} p_{t, t}^{e}\right] .
$$

Eq. (5) describes the term structure of cross-sectional dispersion of expectations conditionally on the rule given by eq. (4).

\footnotetext{
${ }^{12}$ The Heuristic Switching Model, introduced by Anufriev and Hommes (2012), is an example of a model with heterogeneous extrapolation strengths.

${ }^{13} \mathrm{~A}$ cautionary note is in order here: eq. (4) does not intend to be a precise description of the behavior of the subjects when forming their expectations; instead it should be considered as a simple mathematical formalization helping us to better illustrate our conjecture.

${ }^{14}$ The variance of the coefficients $m_{i}$ does not vanish, since we have assumed heterogeneous priors among subjects.
} 
Eq. (5) shows that the dispersion of short-run predictions reflects the subjects' heterogeneity regarding their priors. The linear extrapolation rule amplifies quadratically the heterogeneity of priors across subjects, and therefore, an observed high degree of coordination of short-run predictions $(k=0)$ should not be directly interpreted as a sign of homogeneous expectations regarding future price dynamics. Our simple example shows that the term structure of predictions provides us with crucial information to characterize the heterogeneity of expectations in LtFEs.

We generalize eq. (5) in order to obtain a more flexible expression for describing the term structure of expectations in our experimental data, without necessarily assuming a linear forecasting rule:

$$
\operatorname{Var}\left[{ }_{i} p_{t, t+k}^{e}\right]=(k+1)^{\alpha} \operatorname{Var}\left[{ }_{i} p_{t, t}^{e}\right],
$$

where $\alpha$ represents the shape parameter of the term structure. More precisely, the analysis of the parameter $\alpha$ helps us to have an approximate idea of how much subjects disagree on the evolution of prices as a function of the forecast horizon. In Appendix A, we provide the reader with some simple illustrative examples of the connection between the forecasting rules and the resulting term structure. Given the variance of short-run predictions, $\alpha=0$ means that the level of subjects' disagreement about the future evolution of prices does not depend on the forecast horizon. On the other hand, $\alpha<$ 0 implies that disagreement among subjects about future prices decreases, eventually leading to an agreement on a particular future price level. Finally, $\alpha>0$ implies that disagreement among subjects increases with the forecast horizon: the higher the value of $\alpha$ is, the higher the disagreement of subjects is regarding the future evolution of prices. In particular, for values of $\alpha$ in the range $0<\alpha<1$, the term structure of expectations is concave, signalling a moderate degree of disagreement among subjects. For the edge value $\alpha=1$, the scaling is linear. For values $\alpha>1$, the term structure is convex, which indicates a high degree of disagreement among subjects about future price dynamics. A particular term structure of subjects' disagreement allows us to identify possible candidates for the expectations formation rules used by subjects or discard certain expectations formation rules that are not compatible with a given value of $\alpha$.

In a setting with a positive expectations feedback system, Colasante et al. (2018a,b) show that subjects anchor their short- and long-run expectations to the last realized price ${ }^{15}$, helping the subjects to persistently coordinate

\footnotetext{
${ }^{15}$ Although many contributions in the LtFEs literature have shown that the last realized price constitutes an anchor in the formation of expectations, they limit the forecasting horizon to 1 or 2 -steps ahead predictions.
} 
their short-run expectations. This anchor, however, turns out to be unstable over time because of the typical oscillatory pattern of realized prices in markets characterized by a positive feedback. In the LtFE literature it has been shown that subjects often tend to linearly extrapolate the past observed trend of prices to predict the future price dynamics (see Hommes (2013)). Indeed, the so-called extrapolative bias is a general phenomenon observed in various markets; often economic agents tend to extrapolate or over-extrapolate the observed increasing (decreasing) trend of the market price (see e.g. Barberis et al. (1998), Hirshleifer (2001)). We argue, therefore, that if subjects linearly extrapolate the past trend of prices to form their long-run expectations based on different priors, as in eq. (4), the level of disagreement across subjects increases more than proportionally with the forecast horizon.

Hypothesis 2: Within a positive expectations feedback system, the term structure of predictions exhibits a parabolic shape.

With regards to the negative feedback markets, Heemeijer et al. (2009) have shown that prices exhibit a fast and stable convergence to the fundamental value, despite the lower degree of coordination of short-run expectations as compared to the positive feedback system. Under the hypothesis that subjects form their expectations using an anchor-and-adjustment rule, we argue that stable dynamics of the anchor (namely the fast convergence of the realized price to the fundamental value) can help subjects reduce the uncertainty about the future short-run predictions of other subjects. In other words, it becomes easier for the subjects to guesstimate in period $t$ the other subjects' short-run predictions at the beginning of period $t+k$. Subjects, therefore, learn to anchor their short- as well as long-run predictions to the fundamental value and, consequently, we expect the degree of subjects disagreement to be rather stable for different forecast horizons. This implies that the shape parameter of the term structure is significantly lower than 1 .

Hypothesis 3: Within a negative expectations feedback system, the term structure of predictions exhibits a concave shape.

\section{Results}

Figures (1) and (2) show the dynamics of individual short-run predictions and realized prices for the seven groups with positive feedback and the eight groups with negative feedback. Figures (3) and (4) illustrate, as examples, 
the evolution over time of the price together with individual long-run predictions in one of the groups in the positive and negative feedback treatment, respectively. The other groups show similar patterns.

\subsection{Analysis of short-run predictions}

What Figures 1 and 2 clearly show is that, if the two expectations feedback systems are compared, there are remarkably different patterns in the realized prices. In the positive feedback treatment prices do not show a clear convergence to the fundamental value. While in some groups prices exhibit some kind of monotonic trend towards the fundamental value, in other groups a diverging trend can be observed. Concerning individual short-run predictions, we observe that individual predictions coordinate after some initial periods of volatility, although not necessarily around the fundamental value. On the contrary, in the negative feedback treatment, realized prices converge to the fundamental value after only a few periods, although it does take longer for subjects' short-run predictions to coordinate.

Differences in the convergence of prices to the fundamental value between the positive and negative feedback treatments are quantified in Figure 5, which displays the mean absolute difference between individual short-run predictions and the fundamental value: ${ }^{16}$

$$
M A D_{t, t}^{p_{f}}=\left\langle\frac{\sum_{i=1}^{6}\left|{ }_{i} p_{t, t}^{e}-p_{f}\right|}{6}\right\rangle_{g} .
$$

Figure 5 shows that, although the mean difference decreases in the first five periods, in the markets with positive feedback subjects' predictions do not converge to the fundamental value. A Wilcoxon signed-rank test shows that $M A D_{t, t}^{p_{f}}$ is significantly different from zero in all but the last period. Conversely, in those markets with negative feedback, subjects' predictions converge to the fundamental value. Nevertheless, it takes some time for the price to converge, since it is from period 8 that we observe (by running a Wilcoxon signed-rank test) no statistically significant difference between subjects' predictions and the fundamental value. We also compare the values of $M A D_{t, t}^{p_{f}}$ between treatments and we find that the median distance between predictions and the fundamental value is significantly higher in the positive feedback system compared to the negative feedback system. ${ }^{17}$

\footnotetext{
${ }^{16}$ The notation $\langle\ldots\rangle_{g}$ denotes the average across groups.

${ }^{17}$ We compare treatments by conducting a Wilcoxon signed-rank test whose result confirm that the median value of $M A D_{t, t}^{p_{f}}$ is significantly higher in the positive feedback system $(\mathrm{z}=19.71, \mathrm{p}$-value $<0.001)$
} 
Given our results concerning the convergence of short-run predictions, we can infer that in the positive feedback treatment the REE is not a good benchmark to describe the subjects predictions nor the price dynamics. Our results in the negative feedback treatment show that the individual predictions and prices do converge to the REE. These results are in line with many LtFEs reported in the literature.

Figure 6 quantifies the coordination of subjects' individual short-run predictions measured as the mean absolute deviation between individual 1period-ahead forecasts and the (within-group) average of 1-step-ahead predictions:

$$
M A D_{t, t}^{C}=\left\langle\frac{\sum_{i=1}^{6}\left|{ }_{i} p_{t, t}^{e}-\bar{p}_{t, t}^{e}\right|}{6}\right\rangle_{g} .
$$

In the markets with positive feedback, we observe a fast coordination of subjects' short-run predictions. Similar dynamics are observed in the markets with negative feedback, although it takes longer for short-run predictions to coordinate. ${ }^{18}$ Hypotheses $1 \mathrm{a}$ and $1 \mathrm{~b}$ cannot be rejected by the data. Our results on coordination and convergence of short-run predictions are in line with the literature: while there is fast convergence and slow coordination in the negative feedback treatment, there is slow convergence and fast coordination in the positive feedback treatment.

We now focus on the analysis of individual forecasting behavior to further confirm that our results are consistent with the literature. Following Heemeijer et al. (2009), we estimate the individual prediction strategies of subjects assuming that they use the following linear prediction rule: ${ }^{19}$

$$
{ }_{i} p_{t, t}^{e}={ }_{i} c+\sum_{k=1}^{3}{ }_{i} \alpha_{k} p_{t-k}+\sum_{k=1}^{3}{ }_{i} \beta_{k}{ }_{i} p_{t-k, t-k}^{e}+{ }_{i} \varepsilon_{t} .
$$

We find that eq. (9) provides a good description of the forecasts of 78 out of 90 subjects. ${ }^{20}$ The long-run equilibrium price level is computed as follows:

$$
{ }_{i} \hat{p}=\frac{{ }_{i} \hat{c}}{1-\sum_{k=1}^{3} \hat{\alpha}_{k}-\sum_{k=1}^{3}{ }_{i} \hat{\beta}_{k}},
$$

\footnotetext{
${ }^{18}$ The comparison of $M A D_{t, t}^{C}$ between treatments, by means of Wilcoxon signed-rank test, confirms that they are statistically different. In particular, $M A D_{t, t}^{C}$ in the negative feedback treatment turns out to be significantly higher than that in the positive feedback treatment up to period 10. From the peirod 10 on, the difference between positive and negative feedback treatments is not statistically significant.

${ }^{19}$ We use the same specification of Heemeijer et al. (2009) and have adapted it to our mathematical notation.

${ }^{20}$ We apply the Breusch-Godfrey test for small samples to check the autocorrelation of the residuals.
} 
where ${ }_{i} \hat{c},{ }_{i} \hat{\alpha}_{k}$ and ${ }_{i} \hat{\beta}_{k}$ denote the individual estimates obtained from eq. (9). Figure 7 shows that the distribution of the difference between the long-run equilibrium price $\hat{p}$ and the fundamental value $p_{f}^{21}$ reproduces the main characteristics described in Heemeijer et al. (2009): (i) in markets with negative feedback, subjects are able to learn the REE, given that the distribution of ${ }_{i} \hat{p}-p_{f}$ is highly concentrated around zero; (ii) in markets with positive feedback, long-run equilibrium prices exhibit a higher dispersion around the fundamental value.

Following Heemeijer et al. (2009), since the linear prediction rule is a good descriptor of subjects' forecasts, we estimate for each subject a simpler prediction rule based on the anchor-and-adjustment heuristic. We classify the subjects as naïve, adaptive, trend follower or fundamentalist depending on the individual parameter values. The distribution of subjects among the different categories is similar to that employed by Heemeijer et al. (2009): in the positive feedback treatment roughly $70 \%$ of subjects behave as a trend follower (either naïve or adaptive), whereas in the negative feedback treatment $70 \%$ of subjects behave as a fundamentalist (see the Appendix $\mathrm{C}$ for further details). The empirical analysis on the dynamics of short-run predictions and prices generalizes the results of Colasante et al. (2018a,b) to the case of the negative feedback system. Therefore we can state that eliciting long-run expectations has no significant effect on subjects' short-run predictions, irrespective of the expectations feedback system.

\subsection{Analysis of long-run predictions}

When we first examine Figures 3 and 4, we observe a clear difference in the dynamics of subjects' long-run predictions depending on the implemented feedback system. In the positive feedback treatment, subjects' predictions, although highly coordinated in the short-run around the last realized price, follow a linear trend extrapolation with different slopes, forming a type of cone-shaped trajectory. In contrast, in the negative feedback treatment, the fluctuations of short- as well as long-run predictions exhibit a higher degree of stability fluctuating around the fundamental value.

At first glance, the dynamics of long-run predictions seem to be strongly influenced by the underlying expectations feedback system. In order to quantify whether this is the case for long-run predictions we extend to long-run predictions the analysis on the coordination of subjects' predictions and their

\footnotetext{
${ }^{21}$ Since we have used two different fundamental values, in order to compare the longrun equilibrium price convergence to the fundamental price across markets, we use the difference between the long-run equilibrium price computed using eq. (10) and the corresponding fundamental value.
} 


\begin{tabular}{c|ccc|ccc}
\hline \hline \multirow{2}{*}{$k$} & \multicolumn{3}{|c|}{ Negative Feedback } & \multicolumn{3}{c}{ Positive Feedback } \\
\cline { 2 - 7 } & All periods & period $<10$ & period $\geq 10$ & All periods & period $<10$ & period $\geq 10$ \\
\hline \multirow{2}{*}{$k=2$} & -2.585 & -2.950 & -1.106 & -14.835 & -11.71 & -8.985 \\
& $(0.01)$ & $(0.003)$ & $(0.269)$ & $(0.000)$ & $(0.000)$ & $(0.000)$ \\
& -3.252 & -5.659 & 2.414 & -14.435 & -11.984 & -8.141 \\
\multirow{4}{*}{$k=6$} & $(0.001)$ & $(0.000)$ & $(0.020)$ & $(0.000)$ & $(0.000)$ & $(0.000)$ \\
& -7.578 & -8.421 & 0.237 & -12.382 & -10.4 & -6.69 \\
& $(0.000)$ & $(0.000)$ & $(0.813)$ & $(0.000)$ & $(0.000)$ & $(0.000)$ \\
\hline \hline
\end{tabular}

Table 1: Wilcoxon test on the convergence of $k$-steps-ahead predictions to the fundamental value in the positive and negative feedback treatments. We report the z-values and the p-values in brackets.

convergence to the fundamental value. Figure 8 reports the average convergence of long-run predictions to the fundamental value in the positive and negative feedback treatments, measured as the mean absolute difference between the fundamental value and the predictions submitted in period $t$ for the price in period $t+k$ for different forecast horizons $k$ :

$$
M A D_{t, t+k}^{p_{f}}=\left\langle\frac{\sum_{i=1}^{6}\left|{ }_{i} p_{t, t+k}^{e}-p_{f}\right|}{6}\right\rangle_{g},
$$

where $k=0,1,2,4,6,9$. Figure 8 a shows the lack of convergence to the fundamental value of long-run predictions in the positive feedback treatment. Concerning the negative feedback treatment, Figure 8b shows that convergence significantly improves over time, reaching almost full convergence after period 10. Table 1 confirms these findings. In early periods, the distance from the fundamental value is statistically significant. We can conclude therefore that, under a negative feedback system, subjects learn to converge to the REE, since they coordinate their short and long-run predictions around the fundamental value. On the other hand, under a positive feedback system, subjects' long-run predictions are not well described by the REE.

Figure 9 shows the coordination of subjects predictions measured as the mean absolute deviation between individual $k$-periods-ahead forecasts and their within-group average:

$$
M A D_{t+k}^{C}=\left\langle\frac{\sum_{i=1}^{6}\left|{ }_{i} p_{t, t+k}^{e}-\bar{p}_{t, t+k}^{e}\right|}{6}\right\rangle_{g},
$$

where $k=0,1,2,4,6,9$. 
Subjects learn to coordinate their short and long-run predictions over time. Importantly, for a given period, the cross-sectional dispersion of predictions systematically increases with the forecast horizon, this effect being much more pronounced in the positive feedback treatment. Note that our results regarding coordination of long-run predictions $(k>0)$ are in line with our findings for the 1-step-ahead predictions $(k=0)$.

\subsection{Term structure of the cross-sectional dispersion of subjects' predictions}

In Section 3 we conjectured that the evolution of the dispersion of subjects' predictions over the forecast horizons contains information on the degree of disagreement among subjects. We also suggested that the shape parameter depends on the expectations feedback system. Figure 10 illustrates the evolution of the term structure of the dispersion of predictions across periods and treatments. A first glance at Figure 10 gives the impression that in the positive feedback treatment the slope of the term structure is higher than in the negative feedback treatment.

Let us now analyze the empirical properties of the term structure of expectations of eq. (6). In order to test Hypotheses 2 and 3, we estimate the value of the shape parameter $\alpha$ from a log-linearization of eq. (6) with "normalized" variances, using a pooled panel regression:

$$
\log \left(\frac{<\operatorname{Var}\left[{ }_{i} p_{t, t+k}^{e}\right]>_{g}}{<\operatorname{Var}\left[{ }_{i} p_{t, t}^{e}\right]>_{g}}\right)=\alpha \log (k+1) .
$$

The normalization of the variances allows for a direct comparison of the shape parameter of the term structure between the two treatments. Table 2 shows that the estimated values of $\alpha$ are significantly different from zero in the two treatments. In particular, the case of the positive feedback treatment, the estimated value of the shape parameter $\alpha$ is significantly smaller than 2. This allows us to reject Hypotheses 2, i.e. a parabolic shape of the term structure under a positive expectations feedback system. However, the estimated value of $\alpha$ is significantly higher than 1 , indicating a convex term structure. Althought this might indicate that subjects follow extrapolative rules, these rules are more complex than the simple linear extrapolative rule assumed in Hypothesis 2. On the other hand, in the negative feedback treatment the estimated value of the shape parameter $\alpha$ turns out to be significantly smaller than 1 , which indicates a concave term structure. Thus we cannot reject Hypothesis 3.

We have conjectured that the concave shape of the term structure in the negative feedback treatment is related to the fact that subjects anchor their 


\begin{tabular}{lcc}
\hline \hline & Negative feedback & Positive feedback \\
\hline $\log (k+1)$ & $0.36^{* * *}$ & $1.29^{* * *}$ \\
$\mathrm{SE}$ & $(0.03)$ & $(0.03)$ \\
\hline $\mathrm{N}$ & 117 & 117 \\
$R^{2}$ & 0.56 & 0.91 \\
\hline \hline${ }^{* * *}$ p-value $<0.01,{ }^{* *}$ p-value $<0.05,{ }^{*}$ p-value $<0.1$
\end{tabular}

Table 2: Results of the pooled panel regression from eq. (13). Dependent variable: log of cross-sectional normalized variance of long-run predictions for a given period and forecast horizon. We consider twelve periods $t=2, \ldots, 13$ and ten horizons $k=1, \ldots, 10$ (when possible).

predictions to the past realized price, which exhibits a fast and persistent convergence to the fundamental value. In order to show that the convergence of predictions to the fundamental value reduces the degree of disagreement among subjects over the different forecast horizons, we introduce a similar functional form as in eq. (6) which relates the mean squared deviation of long-run predictions from the fundamental value to the forecast horizons:

$$
\begin{gathered}
M S D_{t, t+k}^{p_{f}}=\left\langle\frac{\sum_{i=1}^{6}\left({ }_{i} p_{t, t+k}^{e}-p_{f}\right)^{2}}{6}\right\rangle_{g}, \\
\operatorname{MSD}_{\mathrm{t}, \mathrm{t}+\mathrm{k}}^{\mathrm{p}_{\mathrm{f}}}=(k+1)^{\mu} \mathrm{MSD}_{\mathrm{t}, \mathrm{t}}^{\mathrm{p}_{\mathrm{f}}} .
\end{gathered}
$$

Next we estimate the corresponding parameter $\mu$ for the positive and negative feedback treatments. ${ }^{22}$ Table 3 shows that the estimated value of the shape parameter $\mu$ is not statistically different from the estimated value of $\alpha$ in the negative feedback treatment. This is consistent with our conjecture that the convergence of predictions to the fundamental value and their mutual coordination are closely related. This close relationship between coordination and convergence of the dynamics does not hold in the positive feedback treatment, and therefore the estimated values for $\mu$ do not provide any relevant information, since long-run predictions do not converge nor coordinate around the fundamental value.

\footnotetext{
${ }^{22}$ We use the same pooled panel regression of the normalized variances as in eq. (13).
} 


\begin{tabular}{lcc}
\hline \hline & Negative feedback & Positive feedback \\
\hline $\log (k+1)$ & $0.37^{* * *}$ & $0.58^{* * *}$ \\
$\mathrm{SE}$ & $(0.03)$ & $(0.03)$ \\
\hline $\mathrm{N}$ & 117 & 117 \\
$R^{2}$ & 0.49 & 0.78 \\
\hline \hline${ }^{* * *}$ p-value $<0.01,{ }^{* *}$ p-value $<0.05,{ }^{*}$ p-value $<0.1$
\end{tabular}

Table 3: Results of the pooled panel regression from eq. (15). Dependent variable: $\log$ of average quadratic difference between individual long-run predictions $p_{t, t+k}^{e}$ and the fundamental value. We consider twelve periods $t=2, \ldots, 13$ and ten forecast horizons $k=1, \ldots, 10$ (when possible).

\section{Conclusion}

We conduct a LtFEs where we simultaneously elicit short and long-run expectations about the evolution of the price in experimental markets characterized by different expectations feedback systems. In particular, we generalize the original contribution of Heemeijer et al. (2009) which elicits short-run expectations and extend the results of Colasante et al. (2018a,b) to markets with a negative expectations feedback system.

Our empirical analysis suggests that, in order to characterize the heterogeneity of expectations it is extremely informative to measure not only the level of dispersion of subjects' expectations, but also to explore how it evolves over different forecasting horizons. We propose a simple term structure model for cross-sectional dispersion of expectations, which is defined by only one parameter, i.e the shape coefficient. The estimated values of the shape coefficient in our experimental data turn out to provide important clues about how the feedback system affects the mechanism of formation of (long-run) expectations. In particular, in the markets with positive feedback the estimated value of the shape coefficient indicates a convex term structure signalling a high level of disagreement among forecasters. This is compatible with an (heterogeneous) extrapolative trend behavior of subjects when forming their expectations. On the other hand, a concave shape characterizes the term structure in the markets with negative feedback, pointing to a smoother evolution of forecasters disagreement. We argue that this behavior is a consequence of the stable convergence of the price to the fundamental value, which helps the subjects predict more accurately the behavior of other subjects and, therefore, the dynamics of future prices.

Concerning the dynamics of prices and expectations, our results reveal 
that eliciting long-run expectations does not influence subjects' short-run expectations. In fact we observe the same aggregate patterns of coordination and convergence of short-run expectations, as well as price dynamics, reported in the LtFEs literature. When extending the time spectrum of subjects' expectations, we find that in the negative feedback markets, subjects learn the REE, since they coordinate their short and long-run expectations around the fundamental value. After a learning phase the REE turns out to be a good predictor of the dynamics of expectations irrespective of the forecast horizon. Conversely, in the positive feedback markets, the REE does not predict the evolution of subjects' expectations, in the short- nor in the long-run. Instead, subjects learn to coordinate their short-run expectations around the last realized price, and tend to extrapolate past trend prices when forming their long-run expectations.

From a more methodological perspective, we are firmly convinced that eliciting long-run expectations in the framework of the LtFEs complements the macroeconomic literature based on surveys about the origin of heterogeneity in expectations. Additionally, it can be successfully employed as testbed for studying economic policy measures within diverse macroeconomic scenarios.

\section{Acknowledgement}

The authors are grateful for the funding by the Universitat Jaume I under the project P11B2015-63, and also the funding by the Spanish Ministry of Science and Technology under the project ECO2015-68469-R and the Valencian Regional Government for the financial support under the project AICO/2018/036. We also thank the participants of the 22nd Annual Workshop on Economic Science with Heterogeneous Interacting Agents for their helpful comments to an earlier version of the paper. 


\section{References}

Mikhail Anufriev and Cars Hommes. Evolutionary selection of individual expectations and aggregate outcomes in asset pricing experiments. American Economic Journal: Microeconomics, 4(4):35-64, 2012.

Tiziana Assenza, Peter Heemeijer, Cars Hommes, and Domenica Massaro. Individual Expectations and Aggregate Macro Behavior. Dnb working papers, Netherlands Central Bank, Research Department, May 2011.

Tiziana Assenza, Te Bao, Cars Hommes, and Domenico Massaro. Experiments on expectations in macroeconomics and finance. In Experiments in macroeconomics, pages 11-70. Emerald Group Publishing Limited, 2014.

Te Bao and Li Ding. Nonrecourse mortgage and housing price boom, bust, and rebound. Real Estate Economics, 44(3):584-605, 2016.

Te Bao, John Duffy, and Cars Hommes. Learning, forecasting and optimizing: An experimental study. European Economic Review, 61:186-204, 2013.

Nicholas Barberis, Andrei Shleifer, and Robert Vishny. A model of investor sentiment1. Journal of financial economics, 49(3):307-343, 1998.

Annarita Colasante, Simone Alfarano, Eva Camacho, and Mauro Gallegati. Long-run expectations in a learning-to-forecast experiment. Applied Economics Letters, 25(10):681-687, 2018a.

Annarita Colasante, Simone Alfarano, Eva Camacho-Cuena, and Mauro Gallegati. Long-run expectations in a Learning-to-Forecast Experiment: A Simulation Approach. Journal of Evolutionary Economics, 2018b. doi: 10.1007/s00191-018-0585-1.

Camille Cornand and Cheick Kader M'baye. Does inflation targeting matter? an experimental investigation. Macroeconomic Dynamics, pages 1-40, 2016.

John Haltiwanger and Michael Waldman. Limited rationality and strategic complements: the implications for macroeconomics. The Quarterly Journal of Economics, pages 463-483, 1989.

Ernan Haruvy, Yaron Lahav, and Charles N Noussair. Traders' expectations in asset markets: experimental evidence. The American Economic Review, 97(5):1901-1920, 2007. 
Peter Heemeijer, Cars Hommes, Joep Sonnemans, and Jan Tuinstra. Price stability and volatility in markets with positive and negative expectations feedback: An experimental investigation. Journal of Economic Dynamics and Control, 33(5):1052-1072, 2009.

David Hirshleifer. Investor psychology and asset pricing. The Journal of Finance, 56(4):1533-1597, 2001.

Cars Hommes. Behavioral rationality and heterogeneous expectations in complex economic systems. Cambridge University Press, 2013.

Cars Hommes, Joep Sonnemans, Jan Tuinstra, and Henk van de Velden. A strategy experiment in dynamic asset pricing. Journal of Economic Dynamics and Control, 29(4):823-843, 2005.

N Gregory Mankiw, Ricardo Reis, and Justin Wolfers. Disagreement about inflation expectations. NBER macroeconomics annual, 18:209-248, 2003.

Charles F Manski. Measuring expectations. Econometrica, 72(5):1329-1376, 2004.

Ramon Marimon, Stephen E Spear, and Shyam Sunder. Expectationally driven market volatility: an experimental study. Journal of Economic Theory, 61(1):74-103, 1993.

Andrew J Patton and Allan Timmermann. Why do forecasters disagree? lessons from the term structure of cross-sectional dispersion. Journal of Monetary Economics, 57(7):803-820, 2010. 


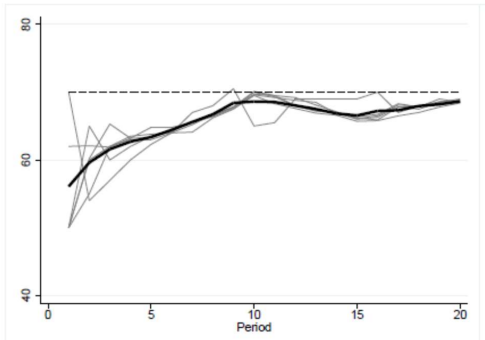

(a) Group 1

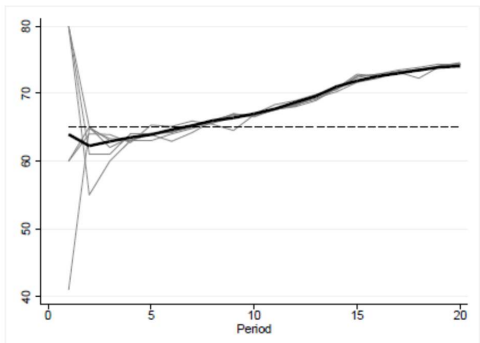

(c) Group 3

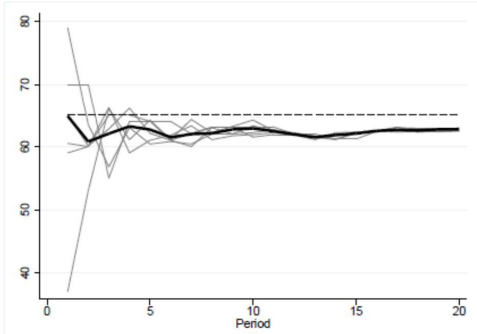

(e) Group 5

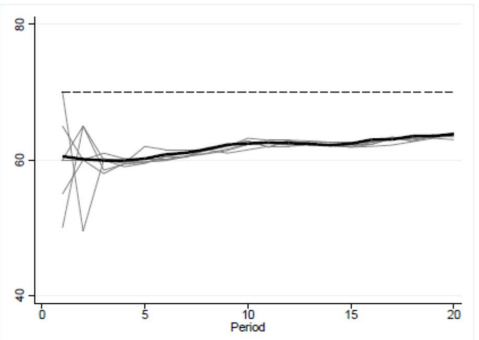

(b) Group 2

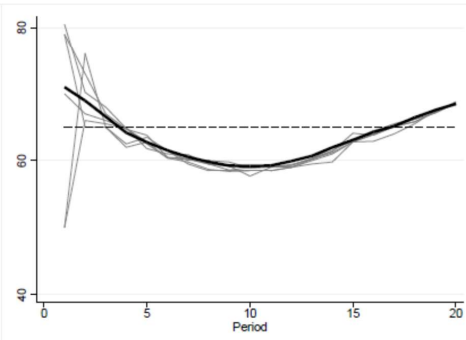

(d) Group 4

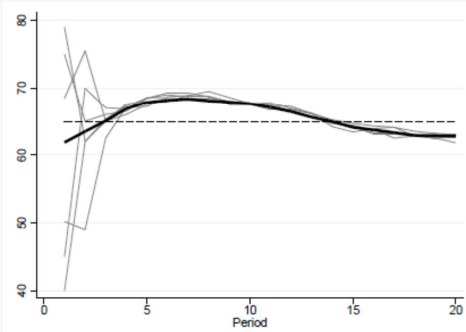

(f) Group 6

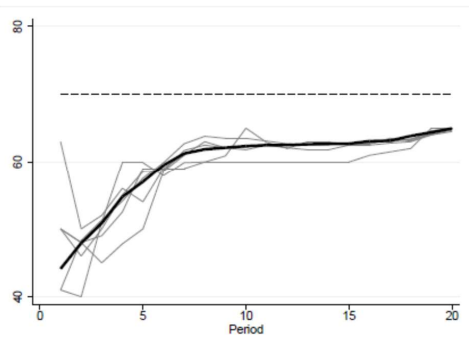

(g) Group 7

Figure 1: Realized price and individual short-run predictions of all groups in the positive feedback treatment. The black solid line represents the realized price, the grey lines are the individual one-step-ahead predictions and the dashed line represents the fundamental value. In groups 1, 2 and 7 $p_{f}=70$, while in groups $3,4,5$ and $6 p_{f}=65$. 


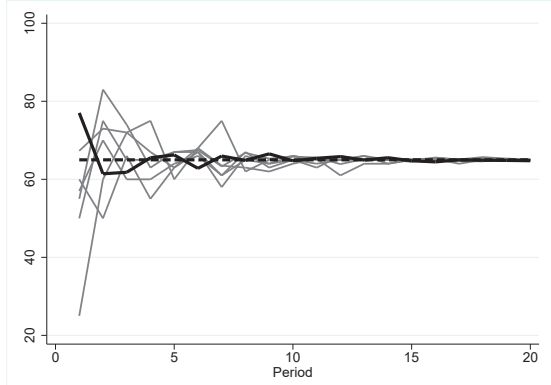

(a) Group 1

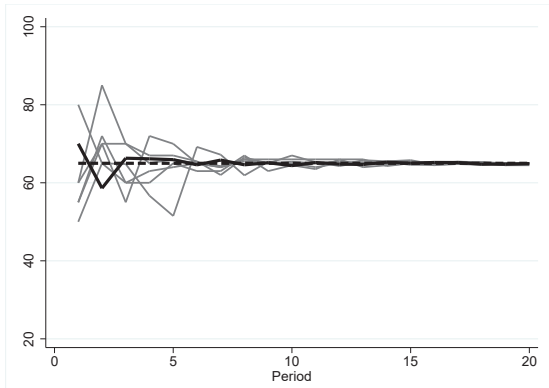

(c) Group 3

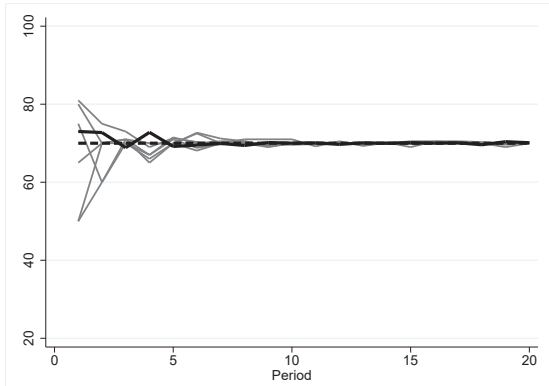

(e) Group 5

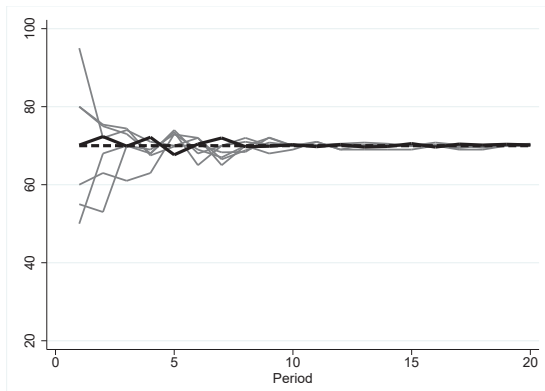

(g) Group 7

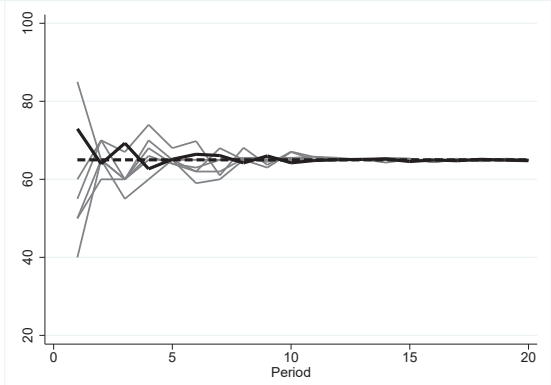

(b) Group 2

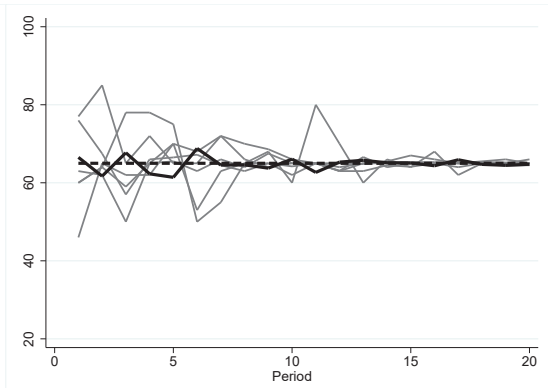

(d) Group 4

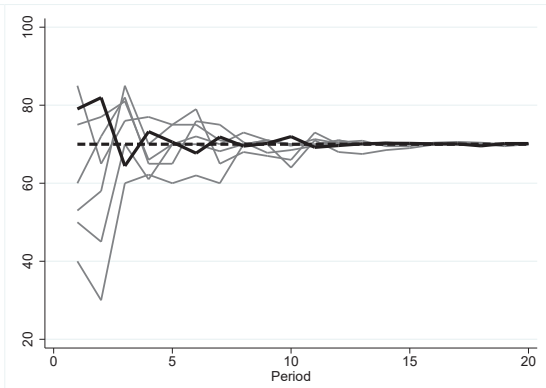

(f) Group 6

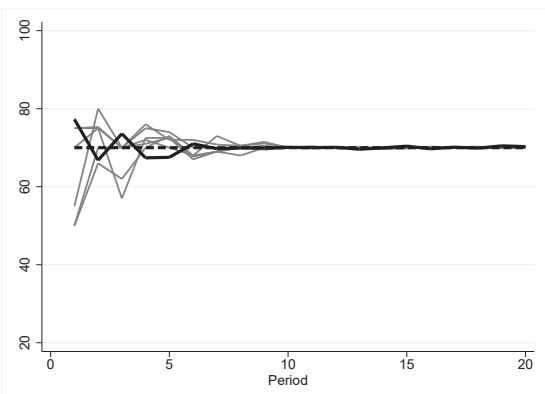

(h) Group 8

Figure 2: Realized price and individual short-run predictions of all groups in the negative feedback treatment. The black solid line represents the realized price, the grey lines are the individual one-step-ahead predictions and the dashed line represents the fundamental value. In groups 1, 2, 3 and $4 p_{f}=65$, while in groups $4,5,6,7$ and $8 p_{f}=70$. 


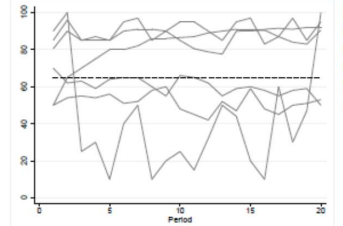

(a) Period 1

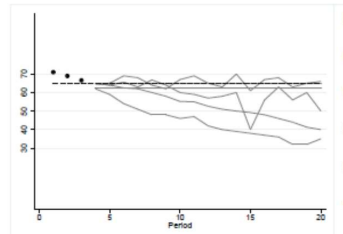

(d) Period 4

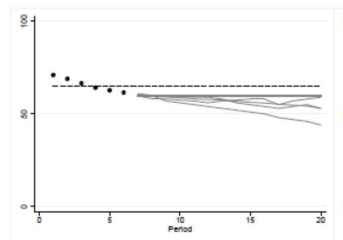

(g) Period 7

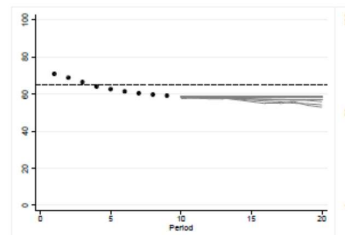

(j) Period 10

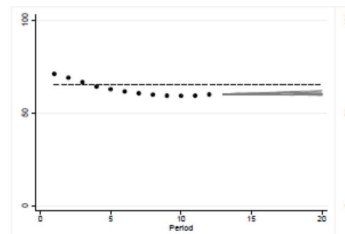

(m) Period 13

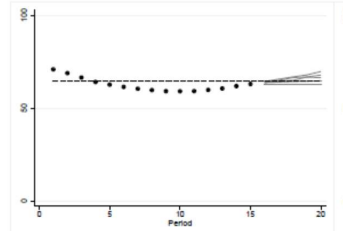

(p) Period 16

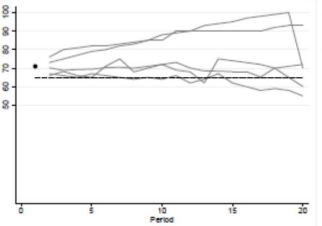

(b) Period 2

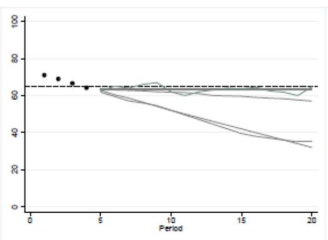

(e) Period 5

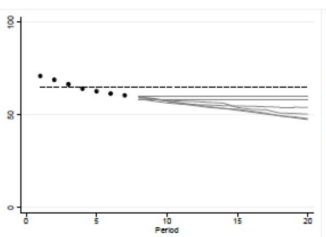

(h) Period 8

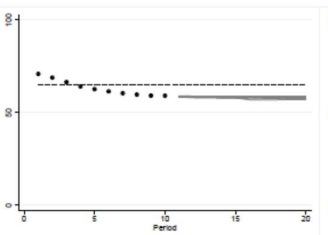

(k) Period 11

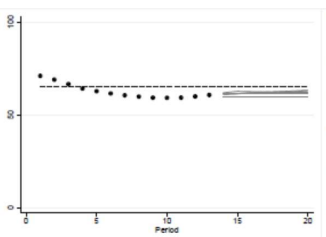

(n) Period 14

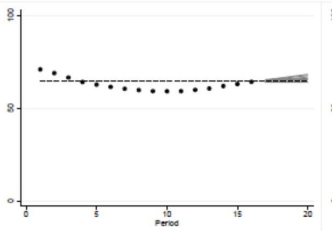

(q) Period 17

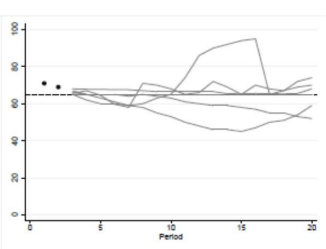

(c) Period 3

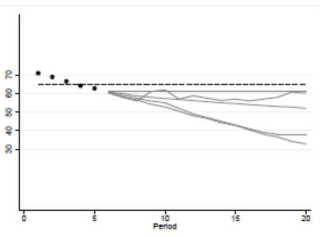

(f) Period 6

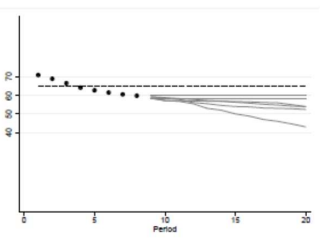

(i) Period 9

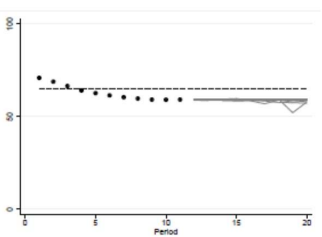

(l) Period 12

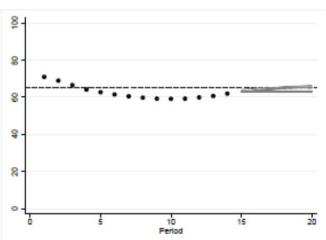

(o) Period 15

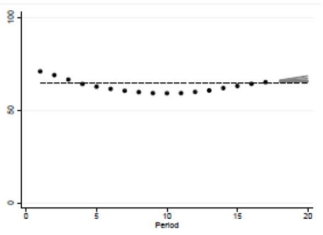

(r) Period 18

Figure 3: Positive feedback:Individual long-run predictions of Group 4. The black dots indicate the realized price, the grey lines the individual forecasts and the dashed line the fundamental value. 


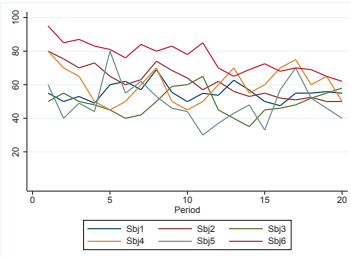

(a) Period 1

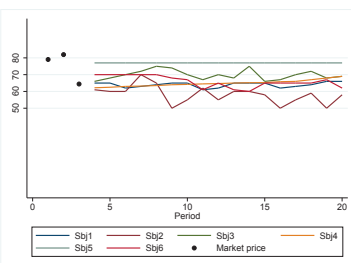

(d) Period 4

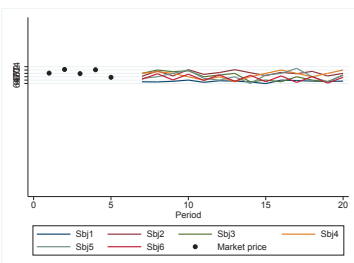

(g) Period 7

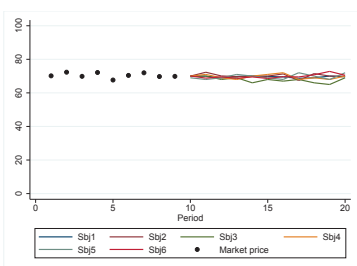

(j) Period 10

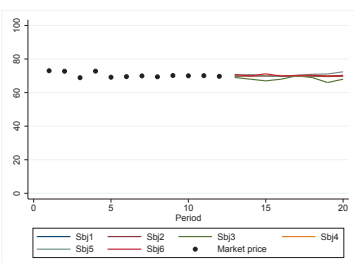

(m) Period 13

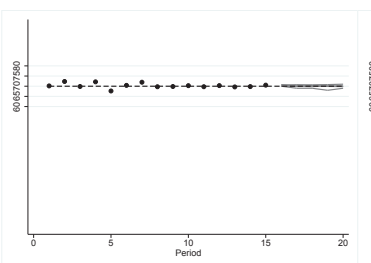

(p) Period 16

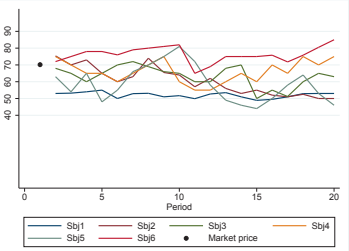

(b) Period 2

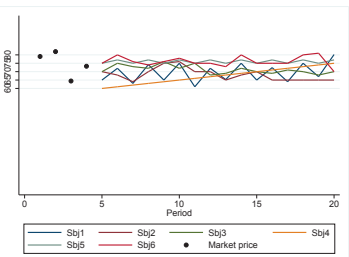

(e) Period 5

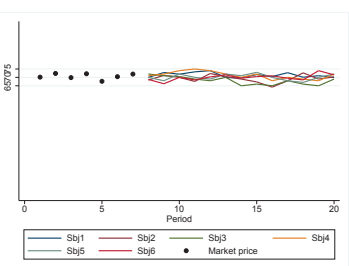

(h) Period 8

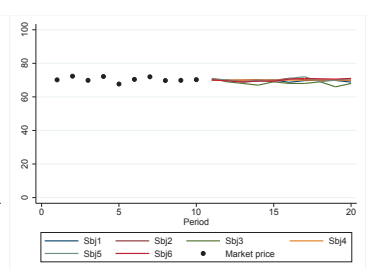

(k) Period 11

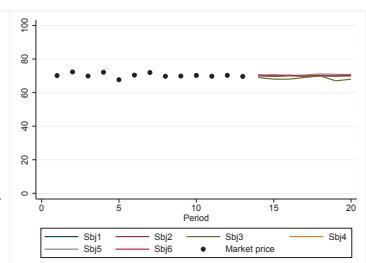

(n) Period 14

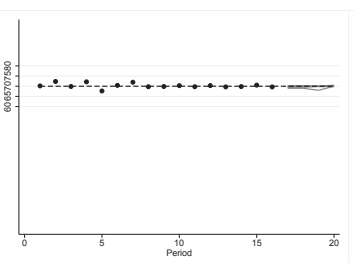

(q) Period 17

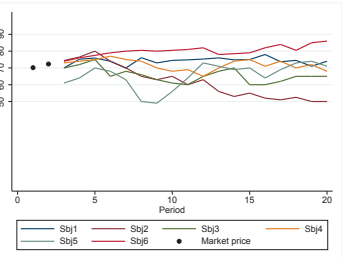

(c) Period 3

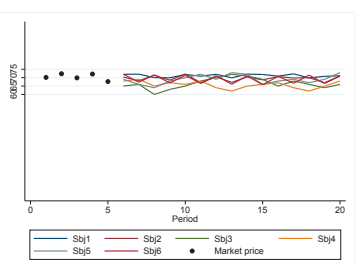

(f) Period 6

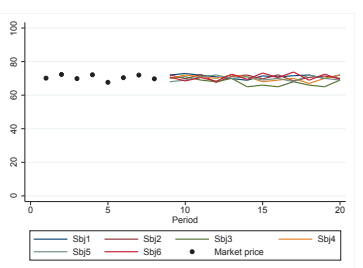

(i) Period 9

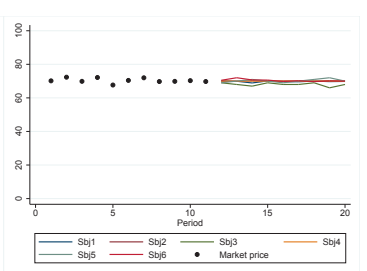

(l) Period 12

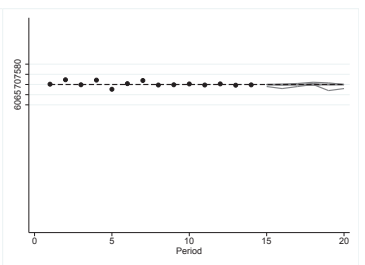

(o) Period 15

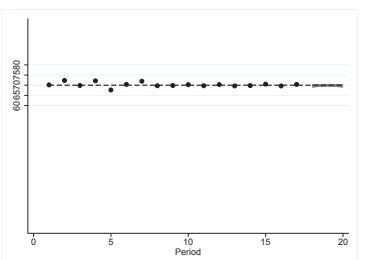

(r) Period 18

Figure 4: Negative feedback: Individual long-run predictions of Group 7. The black dots indicate the realized price, ${ }^{2}$ he grey lines the individual forecasts and the dashed line the fundamental value. 


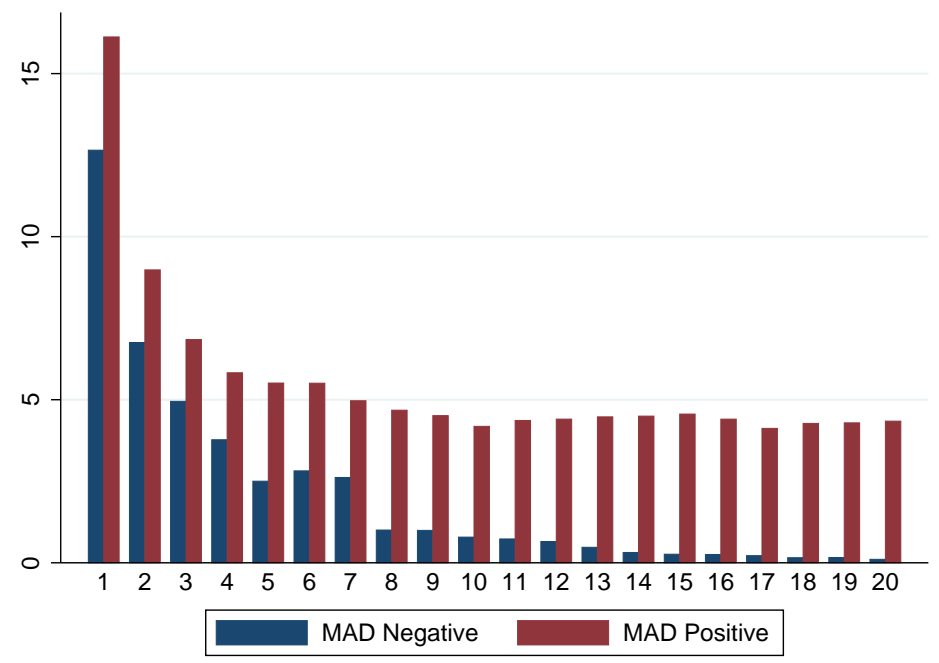

Figure 5: Convergence of short-run predictions: $M A D_{t, t}^{p_{f}}$ in the positive and negative feedback treatments.

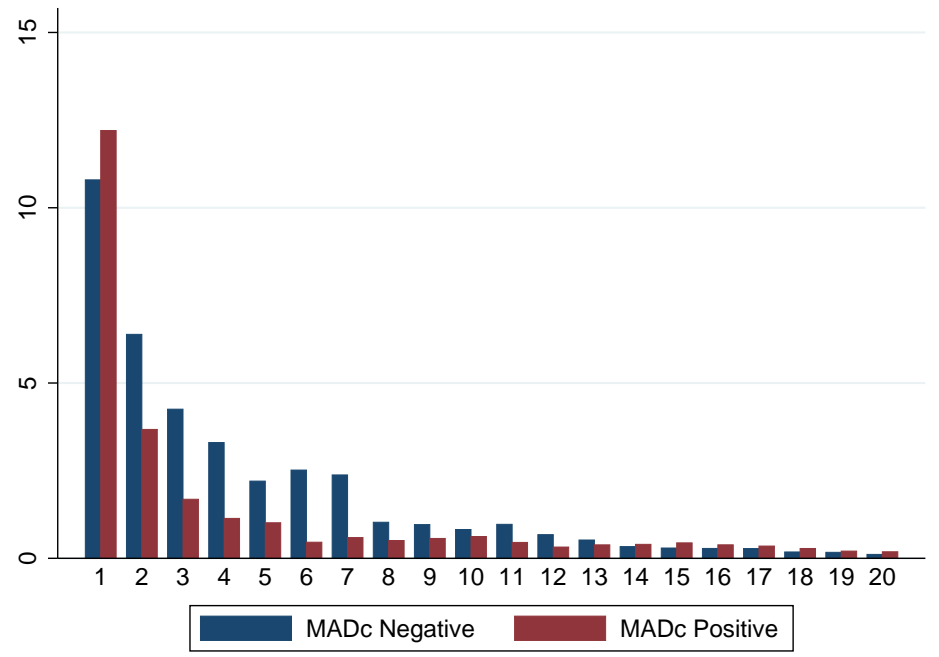

Figure 6: Coordination of short-run predictions: $M A D_{t, t}^{C}$ in the positive and negative feedback treatments. 


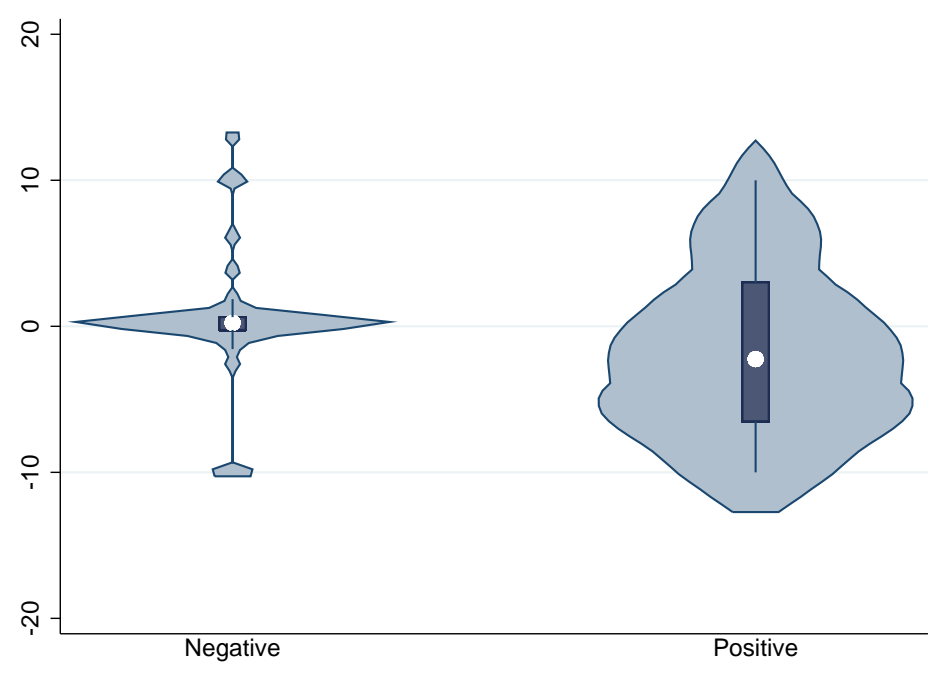

Figure 7: Violin diagram of the distance between the long-run equilibrium price computed following eq. (10) and the fundamental value in the negative and positive feedback treatments. The white dot represents the median value equal to 0.26 and -2.25 for the negative and positive feedback treatment, respectively. In the two treatments a Wilcoxon signed-rank test show no statistically significant difference from zero. 


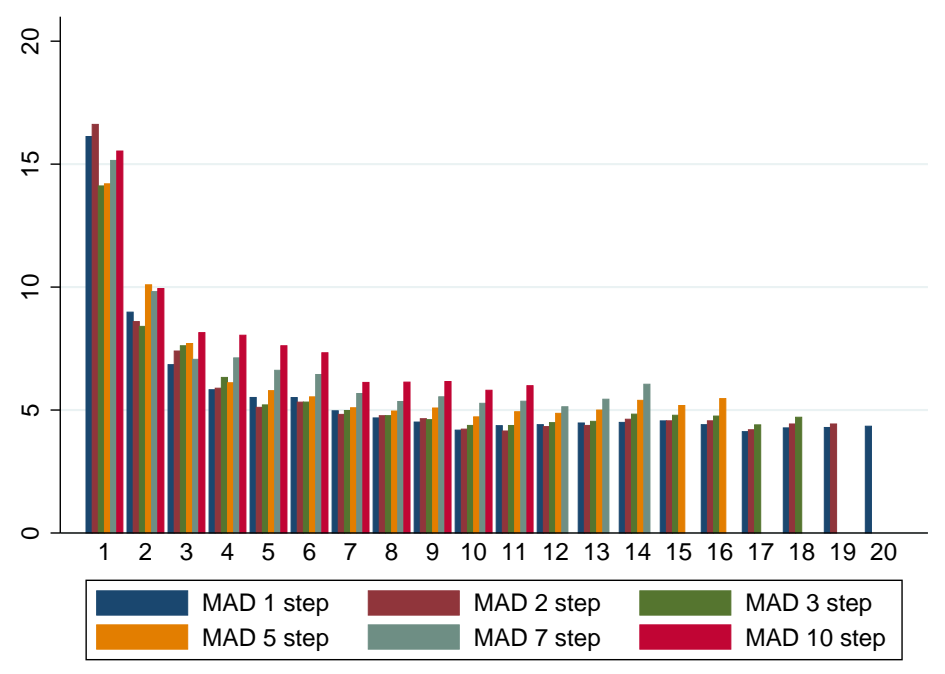

(a) Positive feedback treatment

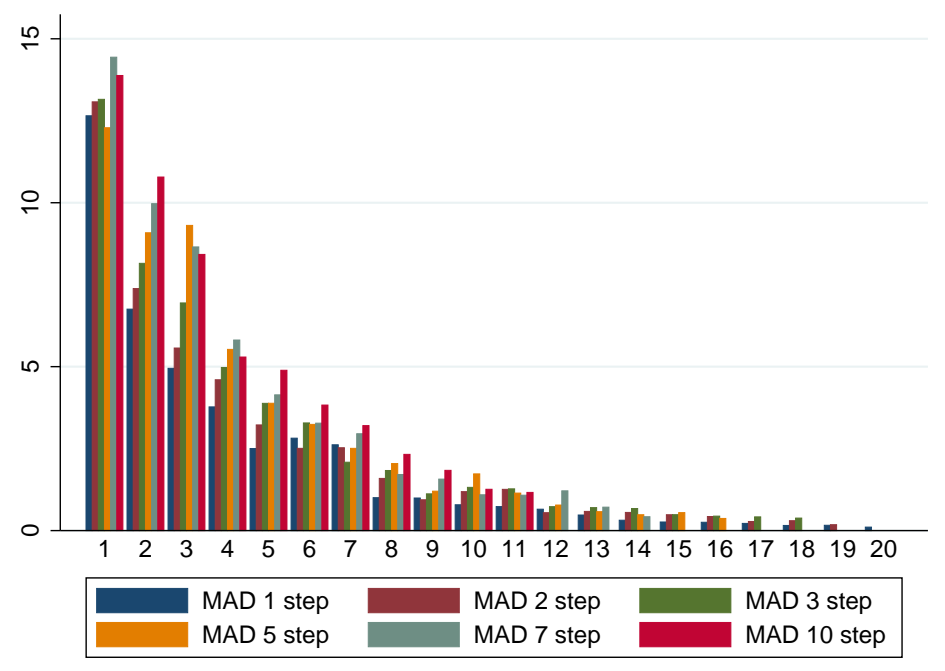

(b) Negative feedback treatment

Figure 8: For each period $t=1, \ldots, 20$, it is displayed the mean absolute deviations between the predictions and the fundamental value for different forecast horizons $k=0,1,2,4,6,9\left(M A D_{t, t+k}^{p_{f}}\right)$, averaged across groups. 


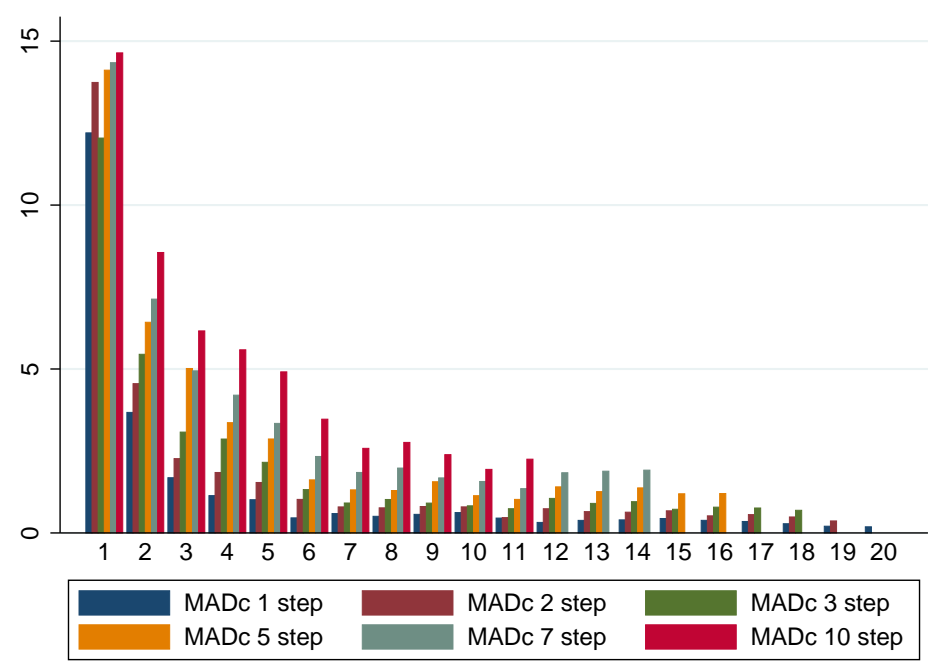

(a) Positive feedback treatment

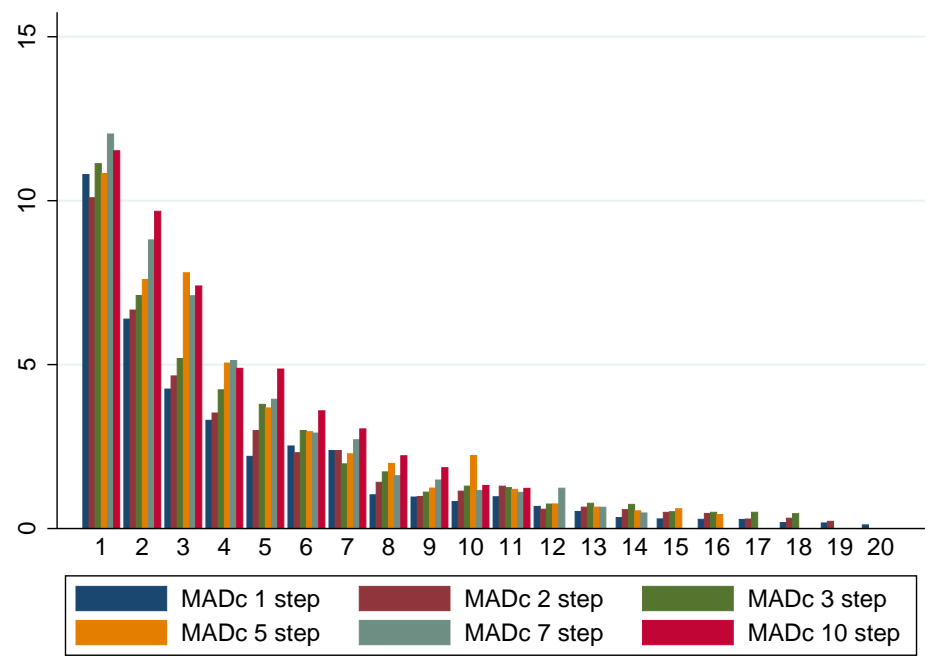

(b) Negative feedback treatment

Figure 9: For each period $t=1, \ldots, 20$ it is displayed the across groups average $M A D_{C}$ of the subjects' forecasts submitted in period $t$ for the price at the end of period $t+k$, where $k=0,1,2,4,6,9$. 


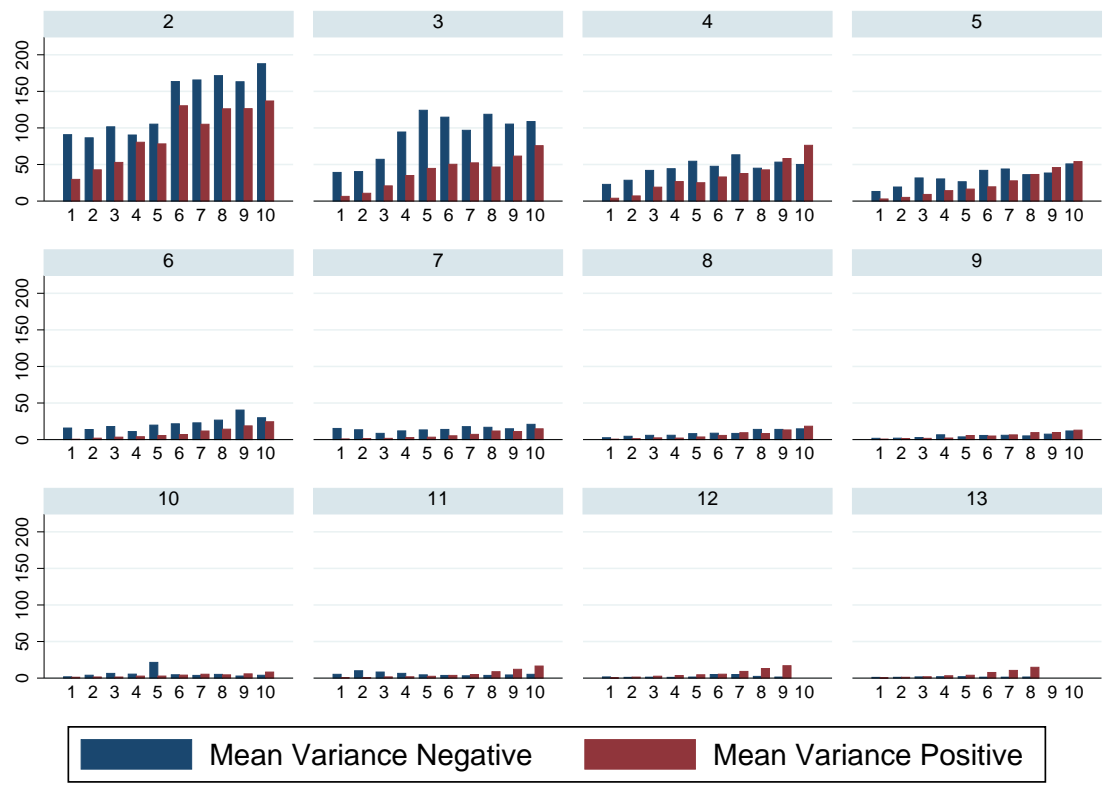

Figure 10: Empirical term structure of the cross-sectional dispersion of expectations: Each histogram displays the variance of the predictions submitted in a given period for different forecast horizons (in the horizontal axis) in the positive and negative feedback treatments. We consider twelve periods $t=2, \ldots, 13$ and ten horizons $k=1, \ldots, 10$ (when possible). 


\section{A Term structure of cross-sectional disper- sion of expectations}

In this appendix we provide some simple examples of how a given forecasting rule affects the term structure of cross-sectional dispersion of expectations.

Let us assume that the subjects, when forming their long run expectations, linearly extrapolate the past price change with a coefficient $m_{i}$ depending on each individual subject. Considering eq. (4), it is possible to compute the average expectation across subjects:

$$
\mathrm{E}\left[i p_{t, t+k}^{e}\right]=p_{t-1}+\mathrm{E}\left[m_{i}\right](k+1)\left(p_{t-1}-p_{t-2}\right) .
$$

The variance of the expectations is therefore:

$$
\operatorname{Var}\left[{ }_{i} p_{t, t+k}^{e}\right]=\operatorname{Var}\left[m_{i}\right](k+1)^{2}\left(p_{t-1}-p_{t-2}\right)^{2} .
$$

Consequently, given that:

$$
\operatorname{Var}\left[{ }_{i} p_{t, t}^{e}\right]=\operatorname{Var}\left[m_{i}\right]\left(p_{t-1}-p_{t-2}\right)^{2}
$$

and plugging it into eq. (17), the term structure is:

$$
\operatorname{Var}\left[{ }_{i} p_{t, t+h+1}^{e}\right]=(k+1)^{2} \operatorname{Var}\left[{ }_{i} p_{t, t}^{e}\right] \text {. }
$$

An alternative to the linear forecasting rule is a "random walk rule", whose starting value is the realized price in the previous period:

$$
{ }_{i} p_{t, t+k}^{e}=p_{t-1}+\sum_{k=0}^{K}{ }_{i} \eta_{t+k}
$$

where ${ }_{i} \eta_{t+k}$ are iid random variables with $\mathrm{E}\left[{ }_{i} \eta_{t+k}\right]=0$ for each $k$ and $i$. Under the rule of eq. (20), it is easy to show that the term structure of the variance is linear in the forecasting horizon:

$$
\operatorname{Var}\left[{ }_{i} p_{t, t+h+1}^{e}\right]=(k+1) \operatorname{Var}\left[{ }_{i} p_{t, t}^{e}\right] \text {. }
$$

An additional possible forecasting rule for long-run predictions is the following:

$$
{ }_{i} p_{t, t+k}^{e}=p_{t-1}+{ }_{i} \xi_{t+k}
$$


where $\xi_{t}$ are iid random variables with $\mathrm{E}\left[{ }_{i} \xi_{t}\right]=0$ for each $t$ and $i$. The variance of the subjects' predictions will not depend on the forecasting horizon $H$ :

$$
\operatorname{Var}\left[{ }_{i} p_{t, t+k+1}^{e}\right]=(k+1)^{0} \operatorname{Var}\left[{ }_{i} p_{t, t}^{e}\right]=\operatorname{Var}\left[{ }_{i} p_{t, t}^{e}\right]
$$

A more general term structure for the dispersion of the predictions exhibits a curvature which depends on the shape parameter $\alpha$ :

$$
\operatorname{Var}\left[{ }_{i} p_{t, t+h+1}^{e}\right]=(k+1)^{\alpha} \operatorname{Var}\left[{ }_{i} p_{t, t}^{e}\right]
$$

which nests all the previous specific cases.

These simple calculations show that the term structure of the variance of subjects' predictions can be extremely informative about the possible underlying rules for the formation of long-run expectations. 


\section{B Instructions and Screenshot}

\section{B.1 Translated instructions (from the original in Spanish)}

\section{[General instructions]}

Welcome to the Laboratory of Experimental Economics! You are participating in an experiment in which you will take decisions in a financial market. The instructions are very simple but, please, read them carefully.

During the whole experiment you will play with experimental currency unit (ECU) and, at the end of the experiment, your final profit, which will be added to a show-up fee of 3 euros, will be converted into euro according to the following exchange rate: 1 Euro=500 ECU. The total amount will be paid at the end of the experiment in cash.

\section{[Only in the positive feedback treatment]}

You are a financial advisor to a pension fund that wants to invest an amount of money to buy an asset. The pension fund has to choose between investing its money in a bank account which pays fixed interest rate and a risky asset that pays dividends. The allocation depends on your forecast for the evolution of the asset price. When making your predictions remember that the asset price in each period is affected as follows: positively by the dividend, negatively by the interest rate and positively by the investors' expectations of the asset price in that period.

Your task is to predict the price for 20 periods. In each period (t) you will predict the price for all the remaining $20-t$ periods, i.e. in period 1 you will submit 20 predictions starting from the prediction about the price at the end of period 1, in period 2 you will submit 19 predictions and so on. Your predictions must be between 0 and 100 .

In period 1 you will submit predictions with information only about the interest rate and the average dividend. From period 2 onwards, you will have more information: a graph with the time series of your past predictions and the time series of the market prices. The green dots represent the time series of your 1-step-ahead predictions, while the blue dots represent the asset price in each period. Additionally, you will see the values of these time series and the time series of all your past predictions. Remember that in any period you will see the information about the asset price in the previous periods. 
The interest rate will be equal to $5 \%$ and the mean dividend will be equal to 3.25 (or 3.5 depending on the session).

\section{[Only in the negative feedback treatment]}

You are an advisor to a firm that wants to buy a certain amount of a particular good. In each period, the manager of the firm decides how many units of this particular good she wants to buy with the aim of selling it in the next period. To take an optimal decision, the manager needs a good prediction of the market price in the next period. The evolution of the market price will be as follows: if the demand for the good is higher than the supply, the price will rise. Conversely, if the supply is higher than the demand, the price will decrease. The manager will take his/her decision based on your predictions about the market price in given period: the higher (lower) the prediction is, the higher (lower) the demand will be and, as a consequence, the market price will fall (rise).

Your task is to predict the price for 20 periods. In each period (t) you will predict the price for all the remaining 20 -t periods, i.e. in period 1 you will submit 20 predictions starting from the prediction about the price at the end of period 1, in period 2 you will submit 19 predictions and so on. Your predictions must be between 0 and 100 .

In period 1 you will submit predictions without any information about past prices. From period 2 onwards, you will see a graph with the time series of your past predictions and the time series of the prices. The green dots represent the time series of your 1-step-ahead predictions, while the blue dots represent the market price in each period. Additionally, you will see the values of those time series and the time series of all your past predictions. Remember that in any period you will see the information about the market price of the previous periods.

\section{[General instructions]}

Once each subject has submitted his/her prediction for each period, the price will be computed and it will be shown at the beginning of period 2 . The same mechanism will be used for subsequent periods. Once you and the other subjects submit the predictions, the price as well as the profits will be computed according to the forecasting accuracy. 
Remember that your profit depends on your forecasting accuracy. The lower your forecasting error (the distance between your predictions and the price in a given period), the higher your profit will be. Your profit will be computed at the end of each period. In addition to the profit for your 1step-ahead prediction each period, you will receive an extra profit for your past predictions about the price for each period. This extra profit will be computed according to the following table:

\begin{tabular}{|c|c|}
\hline $\begin{array}{c}\text { Difference between price of } \\
\text { period } t \text { and your prediction for } \\
\text { period } t\end{array}$ & ECU \\
\hline \pm 5 & 25 \\
\hline \pm 10 & 12 \\
\hline \pm 15 & 5 \\
\hline
\end{tabular}

At the beginning of each period you will see the profit for all the predictions and the cumulative gains.

\section{B.2 Screenshot}




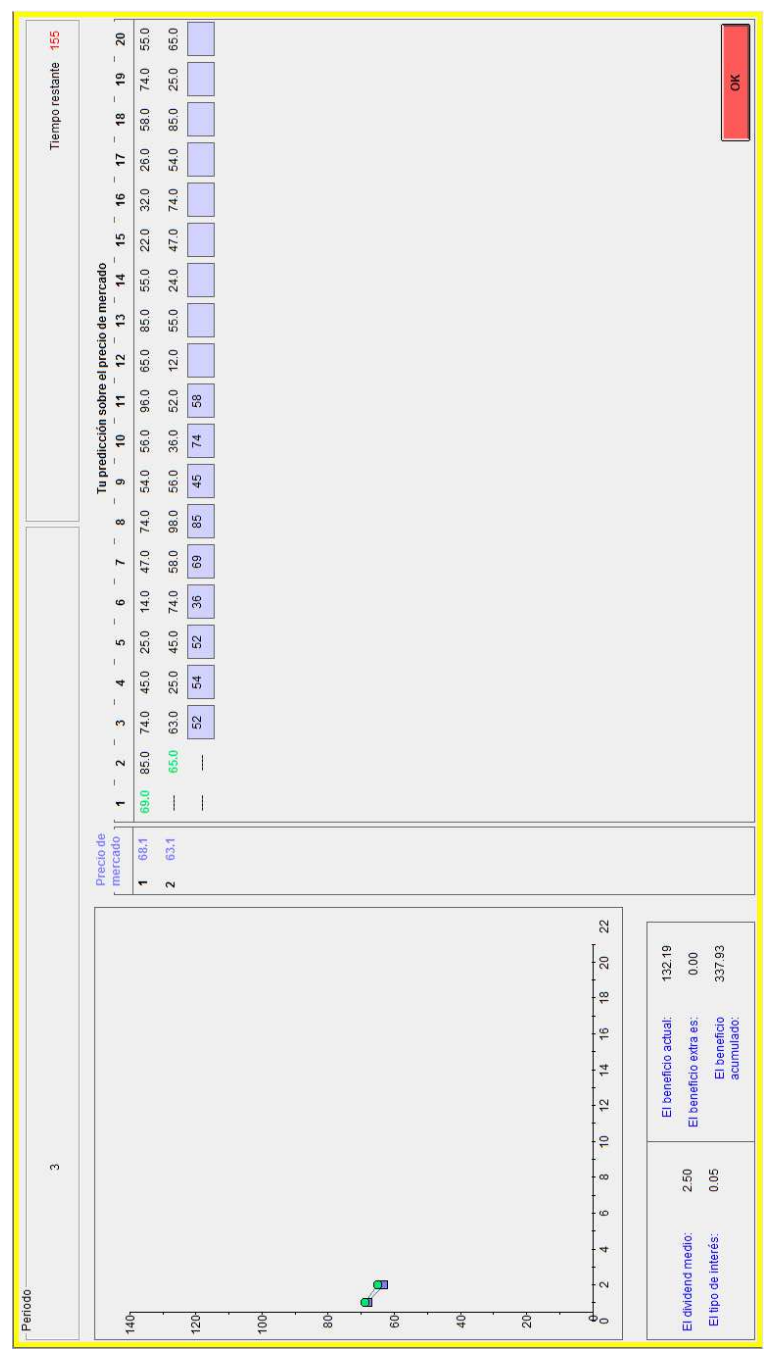

Figure 11: Screen-shot of the experiment. 


\section{Individual prediction strategies}

Following Heemeijer et al. (2009), we estimate the linerar forecasting rule for each subject:

$$
{ }_{i} p_{t, t}^{e}=\alpha_{1} p_{t-1}+\alpha_{2}{ }_{i} p_{t-1, t-1}^{e}+\left(1-\alpha_{1}-\alpha_{2}\right) p_{f}+\beta\left(p_{t-1}-p_{t-2}\right)+\epsilon_{t}
$$

The heuristic described in eq. (25) can be successfully estimated for 72 out of 90 subjects. Using the estimates obtained we can classify subjects as:

(i) Fundamentalist in the negative feedback treatment: $\alpha_{1}+\alpha_{2} \approx 0$ and $\beta=0$

(ii) Adaptive: $\alpha_{1}+\alpha_{2} \neq 0$ and $\beta=0$

(iii) Naïve: $\alpha_{1} \approx 1$ and $\alpha_{2}=\beta=0$;

(iv) Naïve and fundamentalist: $\alpha_{1}<1$ and $\alpha_{2}=\beta=0$

(v) Adaptive trend follower in the positive feedback treatment: $\alpha_{1} \neq$ $0, \alpha_{2} \neq 0$ and $\beta \neq 0$

(vi) Naïve trend follower in the positive feedback treatment: $\alpha_{1} \neq 0, \alpha_{2}=0$ and $\beta \neq 0$

(vii) Trend follower in the positive feedback treatment: $\alpha_{1}=0, \alpha_{2}=0$

Tables 4 and 5 summarize our results and compare them with those reported in Heemeijer et al. (2009).

\begin{tabular}{lcc}
\hline Prediction strategies & Our results & Heemeijer et al. (2009) \\
\hline Naïve trend follower & $34 \%$ & $38 \%$ \\
Adaptive trend follower & $21 \%$ & $33 \%$ \\
Trend follower & $16 \%$ & - \\
None & $29 \%$ & $29 \%$ \\
\hline Observations & 38 over 42 & 21 over 42 \\
\hline
\end{tabular}

Table 4: Positive feedback treatment: individual prediction strategies according to the estimated parameters in eq.(25). 


\begin{tabular}{lcc}
\hline Prediction strategy & Our results & Heemeijer et al. (2009) \\
\hline Fundamentalist & $36 \%$ & $37 \%$ \\
Nave fundamentalist & $15 \%$ & $32 \%$ \\
Adaptive & $13 \%$ & - \\
Naïve & $8 \%$ & $5 \%$ \\
None & $28 \%$ & $26 \%$ \\
\hline Observations & 34 over 48 & 19 over 36 \\
\hline
\end{tabular}

Table 5: Negative feedback treatment: individual prediction strategies according to the estimated parameters in eq.(25). 